\title{
Krisen und Krisenbearbeitung im Referendariat
}

\author{
Stephanie Günther
}

Online publiziert: 27. Juni 2015

(C) Die Autor(en) 2015. Dieser Artikel ist auf Springerlink.com mit Open Access verfügbar.

\section{Rezension zu:}

Fabian Dietrich (2014): Professionalisierungskrisen im Referendariat. Rekonstruktionen zu Krisen und Krisenbearbeitungen in der zweiten Phase der Lehrerausbildung. Springer VS, Wiesbaden, 510 Seiten, 59,99 €, ISBN 978-3-658-03525-9

Professionalisierungsprozesse pädagogisch Tätiger sind ein genuiner Gegenstand erwachsenenpädagogischer Forschung. Angesichts dessen ist die von Fabian Dietrich als Dissertation an der Universität Hannover vorgelegte Studie zu „Professionalisierungskrisen im Referendariat" anregend und bietet auf theoretischer und methodischer Ebene Anlass zur kritischen Würdigung.

Dietrich untersucht Krisenerleben und Krisenbearbeitungen angehender Lehrerinnen und Lehrer in der zweiten Phase der Lehrerausbildung. Hierzu analysiert er objektiv-hermeneutisch Interaktionsprotokolle aus Fallarbeitsseminaren und nimmt dabei sowohl die Seminare als potenziell bedeutsame Orte der Auseinandersetzung pädagogisch Tätiger mit ihrer eigenen Handlungspraxis in den Blick, als auch die individuellen Professionalisierungsprozesse der angehenden Lehrerinnen und Lehrer.

Im Theoriekapitel der Arbeit (Kap. 3 und 4) differenziert Dietrich zentrale Begrifflichkeiten wie Professionalität und Professionalisierung und umreißt, welche Zielvorstellungen sich bzgl. der Lehrerausbildung aus verschiedenen theoretischen Positionen ergeben. Strukturtheoretische Ansätze fokussieren zentrale Handlungsprobleme pädagogischen Handelns, die den schulischen Handlungsraum im Sinne einer Strukturlogik konstituieren. Die Professionalisierungsbedürftigkeit pädagogischen Handelns ergibt sich hier vor allem aus der Annahme ihrer Nicht-Standardisierbarkeit. Kompetenztheoretische Ansätze hingegen seien von der Idee der Optimierung

S. Günther $(\bowtie)$

Universität Potsdam,

Karl-Liebknecht-Straße 24-25,

14476 Potsdam OT Golm, Deutschland

E-Mail: stephanie.guenther@uni-potsdam.de 
schulischer Vermittlungsprozesse getragen und entwerfen „Professionalität als Machbarkeitsversprechen“ (S. 100), welches eingelöst werden könne, wenn angehende Lehrerinnen und Lehrer sich ausreichend adäquate Kompetenzen aneignen. Mit Rekurs auf Angebots-Nutzungs-Modelle werde in kompetenztheoretisch fundierten Studien zwar die Frage nach den Kontexten und Bedingungen bearbeitet, unter denen Kompetenzen erworben werden, nicht aber die Frage wie sich diese Aneignung auf der Subjektseite vollzieht.

Diesem „blinden Fleck“ der Lehrerkompetenzforschung wendet sich Dietrich zu, indem er mit Bezug auf Oevermanns Begriff der Krise eine strukturtheoretisch fundierte Heuristik entwirft, die Professionalisierungsprozesse als habitusgestützte, eigensinnige Bearbeitungen von Krisen modelliert (Kap. 4.4). Entsprechend liegt der Fokus im 260 Seiten starken empirischen Teil der Arbeit (Kap. 6 und 7) nicht auf der in einigen erwachsenpädagogischen Arbeiten noch immer anzutreffenden Frage, ob die angehenden Lehrerinnen und Lehrer professionell handeln. Dies brächte in theoretischer Hinsicht keine neuen Erkenntnisse, da die Praxis hier nur an den Normativen der Theorie gemessen, nicht aber in ihrer Eigenlogik rekonstruiert werden könnte. Dietrich beschränkt sich vielmehr darauf, Krisen induzierende Momente, Umgangsweisen mit krisenhaften Situationen sowie „transformatorische Momente“ zu rekonstruieren, die sich in den Erzählungen von vier Referendarinnen und Referendaren im Rahmen des untersuchten Fallarbeitsseminars abzeichnen. Diese empirische Ausrichtung macht die Untersuchung so interessant.

Zentrales Ergebnis der gründlichen Fallrekonstruktionen ist, dass diejenigen Vorstellungen über pädagogisches Handeln in die Krise geraten, die von einer rationalen Plan- und Kontrollierbarkeit pädagogischer Situationen ausgehen. Die Referendare und Referendarinnen ringen mit einem für Pädagogik konstitutiven Problem: Einerseits halten sie die notwendige Illusion aufrecht, aus der sich pädagogisches Handeln legitimiert - wer pädagogisch handelt, hat ein Ziel, ein Vermittlungsinteresse, eine Wirkhoffnung - und andererseits scheitern sie im pädagogischen Handeln an eben dieser Illusion. Inwieweit die so strukturierten „Professionalisierungskrisen“ Anlass zur Transformation von Vorstellungen pädagogischen Handelns sind, hänge erstens vom Ausmaß der Differenz zwischen Handlungsproblem und Handlungsweise ab, so ein weiteres Ergebnis der Studie - je größer die Differenz, desto größer der Transformationsdruck. Zweitens sei die Qualität der Krisenerfahrung entscheidend. „Transformative Möglichkeitsräume“ eröffnen sich nur dann, wenn Referendarinnen und Referendaren bewusst wird, dass sie sich selbst im Weg stehen, d. h., dass es ihre Deutungsmuster sind, die eine Bearbeitung der als Krise erlebten Situation verhindern (S. 472 ff.).

Transformative Möglichkeitsräume werden im erwachsenenpädagogischen Diskurs aktuell im Kontext transformativer Lern- und Bildungsprozessforschung verhandelt. Ob der von Dietrich in Anschlag gebrachte Krisenbegriff für die empirische Erforschung von Professionalisierungsprozessen im Besonderen und von Bildungsprozessen im Allgemeinen gewinnbringend ist, ob er Missverständnisse evoziert und damit eine aktuell durchaus prominente Annahme stützt, dass Bildung nur dort stattfinde, wo Selbst- und Weltverhältnisse tiefgreifend transformiert werden, ist von den Leserinnen und Lesern selbst zu beurteilen. Wie gehaltvoll empirische Analysen 
dessen sein können, was wir theoretisch mit dem Begriff der Professionalisierung zu fassen suchen, zeigt die Studie allemal.

Open Access Dieser Artikel wird unter der Creative Commons Namensnennung 4.0 International Lizenz (http://creativecommons.org/licenses/by/4.0/deed.de) veröffentlicht, welche die uneingeschränkte Nutzung, Verbreitung und Wiedergabe für beliebige Zwecke erlaubt, sofern Sie den/die ursprünglichen Autor(en) und die Quelle ordnungsgemäß nennen, einen Link zur Creative Commons Lizenz beifügen und angeben, ob Änderungen vorgenommen wurden. 